\title{
Feasibility of Nanotechnology in the Application of Cancer Therapy
}

\author{
Zhiliang Zhang $^{1}$ \\ ${ }^{1}$ Department of chemical engineering, University of Nottingham, Ningbo, Zhejiang, 315000, China
}

\begin{abstract}
The application of nanotechnology in medicine is one of the hot spots now. In this paper, the tumor treatment has carried on the detailed elaboration, from three major treatments called surgery, radiotherapy and chemotherapy. The past treatments and the existing defects of each methods were illustrated, respectively. Then by referring to the relevant literature, what changes the nanotechnology will exert on the three methods were analyzed. In the end, this article presented some shortages and challenges of nanotechnology. According to the research results, nanotechnology can effectively improve the therapeutic effect of the three methods from the following aspects: Firstly, it can improve the accuracy and safety of surgery; Secondly it can enhance the sensitivity of tumor tissues to radiotherapy; Thirdly, it can improve the therapeutic effect of chemotherapy with nano-drugs. In contrast, the low safety and non-standardization of product quality are the main limitations of this technology.
\end{abstract}

\section{Introduction}

The death rate for five leading types of cancer in the United States from 1999 to 2007 are shown below. The death rates for five cancers were stable during this time. For prostate, breast (female only), colon and pancreatic, the death rates for these cancers remained at 10 to 30 percent. And for lung, the death rate was much higher than others in the graph [1]. The percentage of deaths from malignant neoplasms, both for men and women in the United States, 2004 are also showed as follow. Through observing this graph, lung cancer had the highest death rate from cancer, not only among men $(31.3 \%$ of all cancer deaths among men) but also among women (25.6\% of all cancer deaths among women). The second highest cancer for women was breast cancer (15.3\%) and for men was prostate cancer $(10.1 \%)$. Colon cancer was the third highest cancer of death both for men $(9.4 \%)$ and women $(10.1 \%)$ [2]. All the data show that cancer has a significant impact on people's health. Therefore, the treatment of cancer can be developed more in the future. Traditional treatments for cancer include surgery, radiation and chemotherapy. Although these three methods are indispensable in the treatment of cancer, their adverse effects on patients are still great. For example, the immune level decreased after surgery, and the treatment effect was not complete. People need to introduce a more advanced medical technology - nanotechnology.

Nanotechnology is the science and technology of making substances with single atoms and molecules, studying the properties and applications of materials with structural dimensions ranging from 1 to 100 nanometers.
It has been used in physics, biology, chemistry and medical. For nanotechnology used in medical, drug delivery is one of the projects that researchers focus on in recent years. Nanotechnology used in drug delivery can improve the efficiency of treatment extremely. The style of treatment and efficiency of diagnosis can be improved significantly. The materials used for producing nano-carrier are biocompatible which have little toxicity to people. In addition, the surfaces of nano materials are easy to modify. Different viruses and germs corresponding to different receptors will be eliminated. The surfaces of nano materials are changed simply with receptors to treat specific disease efficiently. For surgery, nanoprobes can also be used to identify cancer cells and tissues by fluorescent labelling. For radiotherapy, radioactive element and oxygen delivery carriers are used to improve the effect of treatment.

This paper mainly expounds the changes of three tumor treatment methods -- surgery, radiotherapy and chemotherapy, compares the traditional tumor treatment methods with the treatment methods after the introduction of nanotechnology, and illustrates the application of nanotechnology in different methods by referring to relevant literatures, presenting the advantages of nanotechnology. Finally, in view of the shortcomings of nanotechnology, corresponding improvement measures and research directions will be proposed.

\section{Surgery}

Traditional main treatments for cancer are surgery, radiotherapy and chemotherapy. Surgery is necessary for cancer treatment and has developed for a long time.

Corresponding author's e-mail: ShiLiShuang@cas-harbour.org 
Surgery is one of the main methods to treat and control cancer. It can be used to prevent, diagnosis and reconstruct cancer disease. Preventive treatment is used to eliminate tissue that can become cancerous. For some early-stage cancer, such as breast and colon, and some advanced cancer, such as testicular cancer. These cancers can be cured through surgery. Surgical resection is also essential for palliative treatment, such as quality of life improved by mastectomy in advanced breast cancer, palliative stomas for malignant bowel disease, and stents are implanted to slow the malignant blockage. For reconstructive surgery, it can be applied in cosmesis after mastectomy and for various head and neck operations.

\section{Radiotherapy and Chemotherapy}

Radiotherapy is another commonly used treatment for cancer. Radiation therapy can effectively relieve the symptoms of physical discomfort, during the treatment, patients generally do not have a lot of pain. Compared with surgery, radiotherapy can avoid the side effects of postoperative infection and reduce the damage to patients' physiological functions. There are four main types of radiotherapy for cancer. Firstly, x-rays produced by conventional X-ray therapy machines, because of their low radiation energy, are used for superficial tumor therapy. The second is gamma-rays produced by the decay of the artificial radioactive isotope cobalt. The third is a medical linear accelerator that produces high-energy X-rays and electron lines. These three types are mainly used outside the body. The fourth is to place the radioactive source directly into the treated tissue or into the body's natural cavity. Chemotherapy is the main treatment in the department of oncology. Chemotherapy drugs can be injected or taken orally and distributed in many parts of the body, directly attacking cancer cells until they die. Chemotherapy can be a good adjuvant to other treatment methods, can significantly improve the effect of tumor treatment, and effectively control the spread and metastasis of cancer cells. The principle is to use the cytotoxicity of chemotherapy drugs to kill tumor cells. It is mainly used for adjuvant therapy before and after surgery or radiotherapy.

\section{Defects in surgical treatment}

Firstly, local metastasis and spread of the tumor during surgery. As a result of cutting or squeezing, tumor cells may fall off during surgery, and these tumor cells may fall into surrounding tissue, causing a local transplant, and forming a potential tumor source. In addition, these fallen tumor cells may be transferred to other sites by blood and lymph, where they can lie dormant. When conditions are appropriate, these latent tumor sources multiply rapidly, causing the recurrence of the tumor. Secondly, after receiving operation, patients' body immunity decreases substantially because of the trauma of the operation. Reduced cellular immunity not only makes tumor cells more easily metastasized, but also weakens the inhibition of the body to the tumor area treated in the early stage, thus leading to tumor recurrence. Thirdly, early metastasis is a problem, and the more malignant the tumor, the greater the probability of early metastasis. If the tumor has spread throughout the body, surgery will not solve the problem. Surgery can only be used for local tumor elimination. Finally, surgery itself can lead to different level of organ damage or complications in the body. These problems will bring a lot of inconvenience to the patient's life and physiology.

For example, a research about risk of thiamine deficiency and Wernicke's encephalopathy after gastrointestinal surgery for cancer have found that cancer patients who undergo gastrointestinal surgery are at certain risk for Wernicke's encephalopathy. In addition, patients should receive prophylactic treatment with thiamine regardless of whether symptoms of thiamine deficiency occur [3]. These additional treatments can bring some unnecessary effects to patients.

\section{Defects in Radiotherapy and Chemotherapy}

For radiotherapy, there are three defects for it. Firstly, radiotherapy has a long cycle. For example, in the absence of surgery, radiotherapy takes about six weeks. If adjuvant radiotherapy is used after surgery, the time is about 5 weeks. For example, surgery to treat breast cancer takes about four hours. Postoperative adjuvant radiotherapy takes about 5 weeks. Patients receiving radiotherapy need to pay attention to their eating habits and nutrition mix so that they can resist a long course of radiation therapy. Secondly, because of the advanced radiotherapy equipment, the cost of radiotherapy is relatively high. Radiotherapy is a long-term treatment, and the general radiotherapy treatment process requires 20-30 times, costing nearly 10,000 yuan. Chemotherapy is generally a one-time payment, generally spent in 2000 to 8000 yuan. Thus, the patients receiving radiotherapy need a certain economic basis. Thirdly, radiotherapy may cause a series of complications and have some toxic and side effects on the patient's body. Especially for patients with advanced tumor, the therapeutic effect of radiotherapy is not good, but it has caused serious negative effects on patients, such as insomnia, loss of appetite. For chemotherapy, there are also three defects for it. Firstly, many cancer cells are insensitive to chemotherapy, such as papillary thyroid carcinoma, pancreatic cancer, primary liver cancer. That means chemotherapeutic therapy is very ineffective. Secondly, there are many commonly used chemotherapeutic drugs, such as cyclophosphamide, daunorubicin, adriamycin. These drugs used in chemotherapy have strong chemical toxicity, the toxic and side effects on the body are obvious. For example, liver and kidney function injury, constipation, vomiting, hair loss and other adverse reactions. Thirdly, chemotherapy has a great negative impact on white blood cells and will destroy the patient's immune function. Because immune function is difficult to repair itself, if the patient has residual tumor cells in the body, the spread speed of tumor cells in the body will be accelerated. 


\section{Application of nanotechnology in cancer therapy}

Table 1. Comparison of the Application of nanotechnology in each cancer treatment

\begin{tabular}{|l|c|c|c|}
\hline \multirow{4}{*}{ Cancer treatments } & Surgical treatment & Radiotherapy & Chemotherapy \\
\hline \multirow{2}{*}{ Applications of nanotechnology } & $\begin{array}{c}\text { Nanomaterials used as } \\
\text { hemostatic measures }\end{array}$ & Oxygen delivery carriers & Nano-drug delivery \\
\cline { 2 - 3 } & system & $\begin{array}{c}\text { Nanoparticles used as } \\
\text { radiosensitizers }\end{array}$ \\
\cline { 2 - 3 } & & $\begin{array}{c}\text { Nanocarriers with radioactive } \\
\text { elements }\end{array}$ & \\
\hline
\end{tabular}

\subsection{Application of nanotechnology in surgical treatment}

Traditional surgical treatment will inevitably cause damage to normal tissues or organs. Among them, massive bleeding during or after operation is one of the harmful factors for patients. Some nanomaterials can be used as quick and efficient hemostatic measures to greatly improve the safety of surgical treatment. In addition, the application of nanometer probe and other technologies also plays an important role in improving the accuracy and therapeutic effect of surgery.

In an article of Alyssa B. Chinen, Chenxia M. Guan. et al related to nanoprobes, nanoparticle probes have been designed to detect cancer biomarkers, cells and tissues by fluorescence [4]. These nanoparticles have the advantages of high surface area to volume ratio, specificity and high affinity. There are two main types of fluorescent detection methods: one relies on nanoparticle probes to bind to markers on the surface of cancer cells, and the other relies on probes to enter cells and detect gene content. But most nanoparticle probes work only by binding to cell surface markers, according to reports. The method, which combines the optical properties of some nanoparticles with different surface chemicals, has been used to design nanoprobes for detecting cancer. These new techniques have improved the sensitivity and selectivity of cancer detection methods [4].

\subsection{Application of nanotechnology in radiotherapy}

Due to the lack of oxygen in tumor blood vessels, hypoxia is easy to occur, resulting in much higher radiotherapy tolerance in tumor sites than in normal tissues, leading to poor therapeutic effect. Using nanotechnology to construct oxygen delivery carriers can effectively solve these problems and thus improve the radiosensitivity of tumor sites. In addition, nanoparticles can also be used as radiosensitizers to improve the effect of radiotherapy.
There are some other ways to apply nanotechnology in radiotherapy. For example, radioactive elements in the body are eliminated in two ways. One is that it can be excreted through the kidneys, and the length of time it takes, depending on the size of the particles. As small molecules, radioactive elements cannot exist in human blood circulation for a long time, thus reducing the efficiency of treatment. The other is elimination by opsonization, an immune process in which large molecules are eliminated by phagocytes. However, by combining the nanocarriers with these radioactive elements, the size of the particles was increased. It reduces the chance that these radioactive elements will be eliminated by biological mechanisms, thereby increasing their duration in the bloodstream. In addition, targeting the second clock biological elimination mechanism. Nanoparticles can also reduce opsonization by using polyethylene glycol on their surfaces. Polyethylene glycol on its surface effectively blocks opsonin from binding to radioactive elements, thus prolonging the retention time of radioactive elements in the body [5].

\subsection{Application of nanotechnology in chemotherapy}

The drug powder or solution is stored in nano-sized particles with nanoparticle as the drug carrier. Nanoparticles carry drugs to different parts of the body, where they interact with specific cells. It can solve or alleviate the problems such as low level of accuracy of treatment, side effects of drugs and low drug delivery rate. Nanocarriers have the advantages of long circulation, invisibility and body stability in the body. It can be absorbed by cells and tissues through capillaries and some obstacles in the body. It can control the release of drugs at specific sites, reduce the dosage of drugs, enhance the therapeutic effect and reduce toxicity. In addition, it can avoid the loss of drug activity and facilitate the storage and transportation of drugs. In recent years, it has been found that nano-drug delivery 
system cannot only improve the level of tumor treatment, but also effectively reduce the degree of drug resistance.

For example, in studies of Yongbing Sun, Wen Ma et al related to hepatocellular carcinoma, nanodrugs have improved the problem of drug of lack concentration [6]. In hepatocellular carcinoma (HCC), there is a shortage of drugs during chemotherapy, and most of the drug molecules required for chemotherapy are usually not delivered to the tumor tissue. Among them, the hydrophobicity, toxicity and bioavailability of drug molecules are the main causes of dose limitation. Nanomedicine cannot only solve the problem of drug delivery, but also act specifically on hepatocellular carcinoma. In general, nanotechnology can improve some of the negative effects of chemotherapy, such as improving pharmacokinetics, biodistribution, accumulation of cytotoxic substances at tumor sites, and evaluating the effectiveness of nano-drug delivery systems. Hepatocellular carcinoma is a kind of highly vascular tissue. Its progression is almost accompanied by different etiologies and abnormal angiogenesis at different stages. Therefore, angiogenesis related molecules such as VEGFR, RAF and EGFR are very useful drug targets. Therefore, hepatocyte specific surface molecules including ASGPR, as well as the high expression of endocytic cell surface receptors in hepatocellular carcinoma, can be significantly distinguished from other tissues. These specific associated genotype and phenotypic changes have been used for targeted diagnosis and treatment of hepatocellular carcinoma. For example, in the case of passive targeting, nano-drugs can reach the tumor through the permeability of tumor blood vessels by enhancing permeability and retention (EPR) effects. By passive targeting, nanoparticles can be delivered to specific sites [6].

\section{Discussion}

For nano drug carrier, nano-drug carriers may cause some physical damage. Physical damage refers to the breakdown of nanoparticles in the body, through the way of physical obstruction, causing damage to the microcirculation of cells, thereby causing cell function problems and a series of adverse reactions. The main reason is that the nanocarrier is not biodegradable, so it is easy to accumulate in human body and move freely, causing physical damage to the body. Biodegradable and biocompatible materials should be selected as far as possible to improve the safety of nanomaterials. Additionally, because the fine excipients required by nano-pharmaceutical preparations are few, the further application of nanotechnology is limited to a large extent. Besides, the quality assurance of nanomaterials and regulatory issues such as standardized production also need to be further improved. The research and development technology required by nanomedicine itself is high and the preparation process is complex, which makes it difficult to produce on a large scale. At the same time, due to the influence of equipment, environmental conditions and other factors, its product quality is difficult to standardize.

The research focus of nanotechnology has gradually shifted from basic research to clinical application research, such as the trials of more than 200 nano-drugs in pre-clinical or clinical research, of which $10 \%$ have been successfully entered into phase I clinical trials. More than 50 nano-drugs have been approved, such as liposomes, polymer nanoparticles, micelles, inorganic nanoparticles and protein nanoparticles [7]. To overcome the research and development barriers of nanotechnology, it still needs the further exploration of front-line scientific and technological personnel. At the same time, how to produce a set of reliable quality, large-scale production of nano-drugs, still need further research. In addition, strengthening the application of biodegradable and biocompatible materials in nanomedicine is one of the keys to improve the safety of nanomaterials.

\section{Conclusion}

In cancer treatment, nanotechnology can be used in surgery, radiotherapy and chemotherapy. In terms of surgery, nanomaterials have greatly improved the accuracy and safety of surgery. In radiotherapy, nanomaterials can improve the radiosensitivity of tumors and improve the therapeutic effect. In chemotherapy, the application of nano-drug carrier can better confirm the drug release location and protect the drug activity, so it can improve the treatment effect. It also reduces the number of drugs used, making them less toxic to the body. However, to improve the safety of nanomaterials, finding nanomaterials with biocompatibility and biodegradability will be one of the following research directions. In addition, in terms of nano-drug carriers, how to produce nano-drugs with reliable quality and unified standards on a large scale also requires further efforts of researchers.

\section{References}

1. Morbidity and Mortality Weekly Report, Vol. 59, No. 39 (October 8, 2010), pp. 1281.

2. Morbidity and Mortality Weekly Report, Vol. 56, No. 38 (September 28, 2007), pp. 1006.

3. A. Restivo, M.G. Carta, et al. Risk of thiamine deficiency and Wernicke's encephalopathy after gastrointestinal surgery for cancer. Support Care Cancer (2016) 24, pp. 77-82. Doi: 10.1007/s00520-015-2748-z.

4. A.B. Chinen, C.M. Guan. et al. Nanoparticle Probes for the Detection of Cancer Biomarkers, Cells, and Tissues by Fluorescence. Chemical Reviews, 2015115(19), 10530-10574. Doi: 10.1021/acs. chemrev.5b00321.

5. Y. Mi, Z. Shao, J. Vang, et al. Application of nanotechnology to cancer radiotherapy. Cancer Nano 7, 11 (2016). 
https://doi-org.ezproxy.nottingham.edu.cn/10.1186/s 12645-016-0024-7.

6. Y. Sun, W. Ma. et al. Cancer nanotechnology:

Enhancing tumor cell response to chemotherapy for hepatocellular carcinoma therapy, Asian Journal of

Pharmaceutical Sciences, Volume 14, Issue 6, 2019, Pages 581-594, ISSN 1818-0876, https://doi.org/10.1016/j.ajps.2019.04.005.

7. C. Wang, Q. Fan, Z. Gu, et al. Advances in the application of nanotechnology in cancer prevention, diagnosis and treatment. Tech review, 2018, 36 (22), pp. 96-107. Doi: 10.3981/j.issn.1000-

7857.2018.22.008. 